\title{
Radiation Effects on Magnetohydrodynamic Free Convective Flow over an Infinite Vertical Plate In a Porous Medium
}

\author{
J. D. Olisa \\ Department of Mathematics and Statistics, University of Port Harcourt, Port Harcourt, Nigeria.
}

\begin{abstract}
An analysis to study the unsteady free convective heat transfer due the combined effects of buoyancy, radiation and transverse magnetic field is carried out for an infinite vertical plate in a porous medium. The dimensionless governing coupled partial differential boundary layer equations are formulated and solved analytically using the Laplace transform technique. Results are compared with previous works and found in agreement. The effects of the parameters that enter the problem are discussed.
\end{abstract}

Keywords: Free convection, heat generation, Laplace transform, magnetohydrodynamic, radiation.

\section{Introduction}

Unsteady magnetohydrodynamic free convective boundary layer flow with heat transfer of electrically conducting fluids find their application in a variety of engineering processes such as the design of cooling systems for electronic devices, in the field of solar energy collection, heat exchangers, cooling of metallic plates and MHD marine propulsion. Convective flow which is as a result of density differences created within the fluid are significant in electronic equipments, flow meters and spacecraft systems. Earlier studies have been carried out by many researchers such as [1] - [4]. Also,[5] considered natural convective flow with suction and mass transfer in a porous medium. [6] examined the natural convection with internal heat generation which decays exponentially. Also the effect of mass transfer and free convection past a vertical porous plate was investigated by [7].

Later,[8] considered the solution for hydro magnetic mixed convection heat and mass transfer flow through a porous media while [9] investigated the mass and heat transfer flows past a vertical plate. Also the flow of an unsteady viscous incompressible electrically conducting fluid along a porous vertical isothermal nonconducting plate with variable suction and exponentially decaying heat generation was investigated by [10]. Amongst others, [11] examined the transient laminar MHD free convective flow with non-uniform heat flux.

In all these investigations, the analysis was carried out numerically, and also radiation effects were neglected. Radiation effects are quite significant in some industrial and space technology applications such as glass production and spacecraft re-entry aerothermodynamics. These operate at high temperatures, such that radiation effect cannot be neglected. Radiation effects when considered complicates the governing equations, but [12] have shown that in the optically thin limit, the physical situation can be simplified. They obtained an exact solution to the problem of a radiating laminar fully developed convective flow in an infinite vertical channel. [13] Showed that the fluid does not absorb its own emitted radiation, for an optically thin limit, but absorbs radiation emitted by the boundaries.

Radiation effects have also been considered numerically by [14], [15] and recently by [16]. Analytically, [17] examined the effect of radiation on unsteady free convective flow are a porous plate, while [18] investigated its effect and chemical reaction on MHD flow over a vertical surface.

In this paper, the effect of radiation on unsteady magnetohydrodynamic free convective flow over an infinite vertical plate in a porous medium with heat generation is examined. The resulting dimensionless governing equations are solved by the Laplace transform technique.

\section{Mathematical Analysis}

Consider the radiative effect on unsteady free convective laminar, boundary layer flow of a viscous, incompressible, fluid along an infinite vertical plate due to buoyancy effects, in a porous medium. The flow is of an electrically conducting, gray gas near equilibrium on an optically thin limit. The $\mathrm{x}$-axis is measured along the plate, a magnetic field of uniform strength $\mathrm{B}_{0}$ is applied in the $\mathrm{y}$ direction normal to the vertical plate. 


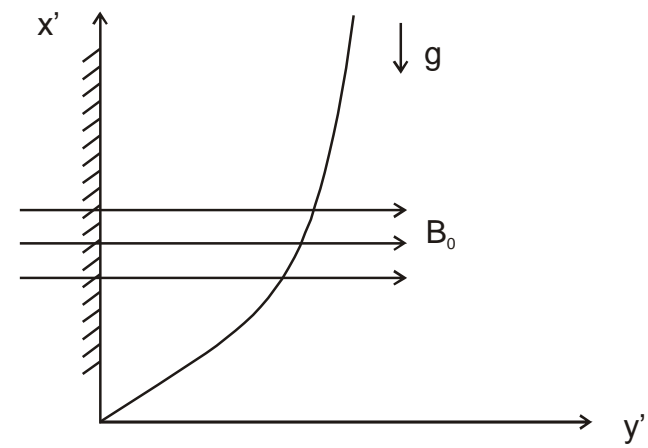

Figure (1) Geometrical configuration of the flow

The applied magnetic field and the magnetic Reynolds number are assumed to be very small, hence the induced magnetic field and the Hall effect are negligible. Since the velocity of the fluid is low, the viscous dissipation is also assumed to be negligible. Since the plate is on the $\mathrm{x}$-axis and is infinite, all the physical quantities depend only on y and t. Then, under the Boussinesq's approximation, the governing equations of the flow are:

$$
\begin{aligned}
\frac{\partial u^{\prime}}{\partial t^{\prime}}= & g \beta\left(T^{\prime}-T_{\infty}\right)-v \frac{\partial^{2} u^{\prime}}{\partial y^{\prime 2}}-\frac{\sigma B_{0}^{2} u^{\prime}}{\rho} \\
- & \frac{v}{K^{\prime}} u^{\prime} \\
& { }_{\rho} C_{p} \frac{\partial T^{\prime}}{\partial t^{\prime}}=k \frac{\partial^{2} T}{\partial y^{\prime 2}}-\frac{\partial q_{r}}{\partial y^{\prime}}+Q_{0}\left(T-T_{\infty}\right)
\end{aligned}
$$

Where $\frac{\partial q_{r}}{\partial y^{1}}$ represents the change in the radiative flux with distance normal to the plate. The radiative heat flux of an optically thin gray gas, using the Rosseland approximation is given by:

$$
\frac{\partial q_{r}}{\partial y^{\prime}}=-4 a * \sigma\left(T_{\infty}^{4}-T^{4}\right)
$$

It is assume that the temperature differences within the flow are sufficiently small such that $T^{4}$ may be expressed as a linear function of the temperature. Hence expanding $T^{4}$ in a Taylor series about $T_{\infty}$ and neglecting higher-order terms:

$$
T^{4} \cong 4 T_{\infty}^{3} T-3 T_{\infty}^{4}
$$

Thus $\frac{\partial q_{r}}{\partial y^{\prime}}=-16 a^{\alpha} \sigma T_{\infty}^{3}\left(T_{\infty}-T\right)$

Equation (2) becomes

$$
\begin{aligned}
\frac{\partial T}{\partial t^{\prime}}= & \frac{K}{\rho C p} \frac{\partial^{2} T}{\partial y^{2}}-\frac{16 a^{*} \sigma T_{\infty}^{3}}{\rho C p}\left(T-T_{\infty}\right) \\
& +\frac{Q_{0}}{\rho C p}\left(T-T_{\infty}\right)
\end{aligned}
$$

The initial and boundary conditions are:

$$
\left.\begin{array}{r}
t^{\prime} \leq 0, u^{\prime}=0, T=T_{\infty} \text { for all } y^{\prime} \\
t^{\prime}>0, u^{\prime}=v_{0}, T=T_{w} \text { at } y^{\prime}=0 \\
u=0, \quad T \rightarrow T_{\infty} \text { as } y^{\prime} \rightarrow \infty
\end{array}\right\}
$$

The following dimensionless quantities are introduced: 
$u=\frac{u^{\prime}}{v_{0}}, y=\frac{y^{\prime} v_{0}}{v}, t=\frac{t^{\prime} v_{0}^{2}}{v}, \Theta=\frac{T-T_{\infty}}{T_{w}-T_{\infty}}$

$G r=\frac{g \beta v\left(T_{w}-T_{\infty}\right)}{v_{0}^{2}}, \operatorname{Pr}=\frac{v \rho C p}{R}, R a=\frac{16 a^{*} v^{2} \sigma T_{\infty}^{3}}{k v_{0}^{2}}$

$M=\frac{\sigma B_{0}^{2} v}{\rho v_{0}^{2}}, K=\frac{k^{\prime} v_{0}^{2}}{v^{2}}, Q=\frac{Q_{0} v}{v_{0}^{2} \rho C p}$

Introducing equation (5) into equations (1) and (3), the dimensionless governing equation are obtained as follows:

$$
\begin{aligned}
& \frac{\partial u}{\partial t}=\frac{\partial^{2} u}{\partial y^{2}}+G r \Theta-\left(M+\frac{1}{K}\right) u \\
& \frac{\partial \Theta}{\partial t}=\frac{1}{\operatorname{Pr}} \frac{\partial^{2} \Theta}{\partial y^{2}}-\left(\frac{R a}{\operatorname{Pr}}-Q\right)
\end{aligned}
$$

The corresponding dimensionless initial and boundary conditions are:

$$
\left.\begin{array}{c}
t \leq 0: u=\Theta=0 \text { for all } y \\
t>0: u=\Theta=1 \text { on } y=0 \\
: u \rightarrow 0, \Theta \rightarrow 0 \text { as } y \rightarrow \infty
\end{array}\right\}
$$

Equations (6) and (7) are coupled non-linear systems of partial differential equations, and are solved analytically by utilizing the Laplace transform technique, subject to equation (8). The transformed ordinary differential equations are:

$$
\frac{d^{2} \bar{u}}{d y^{2}}-(s+a) \bar{u}=G r \bar{\Theta} \quad \text { (9) } \quad \frac{d^{2} \bar{\Theta}}{d y^{2}}-\operatorname{Pr}(s+b) \bar{\Theta}=0
$$

Subject to

$$
\left.\begin{array}{l}
\bar{u}=\bar{\Theta}=\frac{1}{s} \text { on } y=0 \\
\bar{u} \rightarrow 0_{1} \bar{\Theta} \rightarrow 0 \text { as } y \rightarrow \infty
\end{array}\right\}
$$

Where $a=M+\frac{1}{K}, b=\frac{R a}{\operatorname{Pr}}-Q$ and $\mathrm{s}$ is the transformed variable. The solutions of equations (9) and (10) subject to (11) are:

$$
\begin{aligned}
\bar{\Theta}= & \frac{1}{s} e^{-y \sqrt{\operatorname{Pr}(s+b)}} \\
\bar{u}= & \left\{\frac{1}{s}-\frac{G r}{s[(1-\operatorname{Pr}) s-(\operatorname{Pr} b+a)]}\right\} e^{-y \sqrt{s+a}} \\
& +\frac{G r e^{-y \sqrt{\operatorname{Pr}(s+b)}}}{s[(1-\operatorname{Pr}) s-(\operatorname{Pr} b+a)]}
\end{aligned}
$$

Employing the shifting and convolution theorems and [19] equations [12] and [13] are inverted to obtain the solutions:

$$
\begin{aligned}
\theta & =\frac{1}{2}\left\{e^{-y \sqrt{a}} \operatorname{erfc}\left(\frac{y \sqrt{\mathrm{Pr}}}{2 \sqrt{t}}-\sqrt{b t}\right)\right. \\
& \left.+e^{y \sqrt{\mathrm{Pr} b}} \operatorname{erfc}\left(\frac{y \sqrt{\mathrm{Pr}}}{2 \sqrt{t}}-\sqrt{b t}\right)\right\}
\end{aligned}
$$


$u=\frac{1}{2}\left[\begin{array}{l}e^{-y \sqrt{a}} \operatorname{erfc}\left(\frac{y}{2 \sqrt{t}}-\sqrt{a t}\right) \\ +e^{y \sqrt{a}} \operatorname{erfc}\left(\frac{y}{2 \sqrt{t}}+\sqrt{a t}\right)\end{array}\right]-\frac{G r}{c(1-\operatorname{Pr})}\left\{\frac{1}{\frac{1}{2}}\left[\begin{array}{l}e^{-y \sqrt{a}} \operatorname{erfc}\left(\frac{y}{2 \sqrt{t}}-\sqrt{a t}\right) \\ +e^{y \sqrt{a}} \operatorname{erfc}\left(\frac{y}{2 \sqrt{t}}+\sqrt{a t}\right)\end{array}\right]\right.$

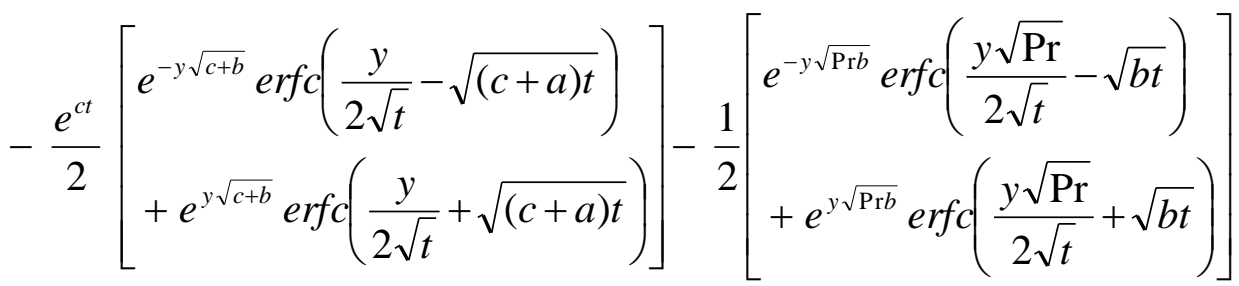

$+\frac{e^{c t}}{2}\left[\begin{array}{l}e^{-y \sqrt{\operatorname{Pr}(c+b)}} \operatorname{erfc}\left(\frac{y \sqrt{\operatorname{Pr}}}{2 \sqrt{t}}-\sqrt{(c+b) t}\right) \\ +e^{y \sqrt{\operatorname{Pr}(c+b)}} \operatorname{erfc}\left(\frac{y \sqrt{\operatorname{Pr}}}{2 \sqrt{t}}+\sqrt{(c+b) t}\right)\end{array}\right]$

Where $c=\frac{\operatorname{Pr} b+a}{1-\operatorname{Pr}}$

Also the viscous stress at the surface of the plate is obtained using

$$
\tau=-\left(\frac{\partial u}{\partial y}\right)_{y=0}
$$

Hence

$$
\begin{aligned}
& \tau=-\left[\sqrt{a}(1-\operatorname{erf}(\sqrt{b t}))-\frac{1}{\sqrt{\pi t}} e^{-a t}\right]+\frac{G r}{c(1-\operatorname{Pr})}\left[\sqrt{a}(1-\operatorname{erf}(\sqrt{a t}))+\frac{1}{\sqrt{\pi t}} e^{-b t}\right] \\
& +e^{c t}\left[\begin{array}{l}
(c+b)(1-\operatorname{erf}(\sqrt{(c+a) t})) \\
+\frac{1}{\sqrt{\pi t}} e^{-(c+a) t}
\end{array}\right]+\left[\sqrt{\operatorname{Pr} b}(1-\operatorname{erf}(\sqrt{b t}))+\sqrt{\frac{\operatorname{Pr}}{\pi t}} e^{-b t}\right] \\
& -e^{c t}\left[\sqrt{\operatorname{Pr}(c+b)}(1-\operatorname{erf} \sqrt{(c+b) t})+\sqrt{\frac{\operatorname{Pr}}{\pi t}} e^{-(c+b) t}\right]
\end{aligned}
$$

\section{Discussion of Results}

The analytical solutions of the dimensionless velocity and temperature equations were obtained using the Laplace transform technique. The analysis of the resulting solutions are carried out by employing the Mathematica software. The consideration is for air for which $\operatorname{Pr}=0.7$, except for the cases where the $\operatorname{Pr}$ is varied for both velocity and temperature profiles.

In Fig. 2 and 3 the effect of $P r$ on both temperature and velocity profiles is shown when $P r$ is varied. The figures show a decrease in the temperature and an increase in the velocity as $\operatorname{Pr}$ increases, when $G r=0.5 R a=$ 0.1 and $Q=2.0 \mathrm{~K}=1.0$ 
Figure 3 depicts the effect of magnetic parameter, $M$ on the velocity and it is observed that the velocity increases as $M$ increases.

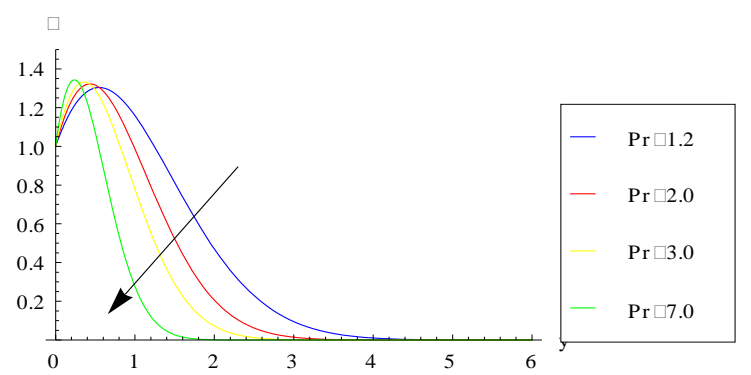

Figure (2) Effect of Pr on temperature profile
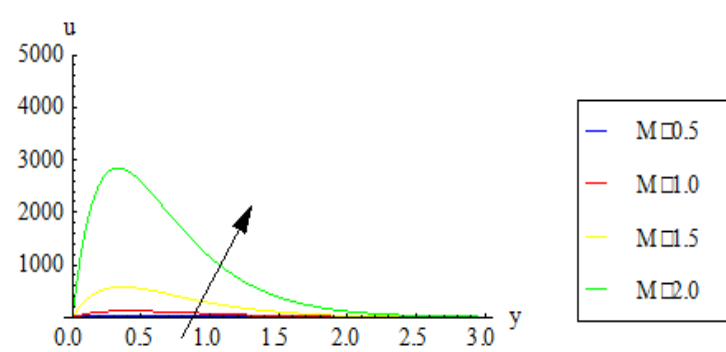

Figure (4) Effect of $\mathrm{M}$ on velocity profiles with $\mathrm{K}$ $=1.0, \operatorname{Pr}=0.7, \mathrm{Q}=2.0$
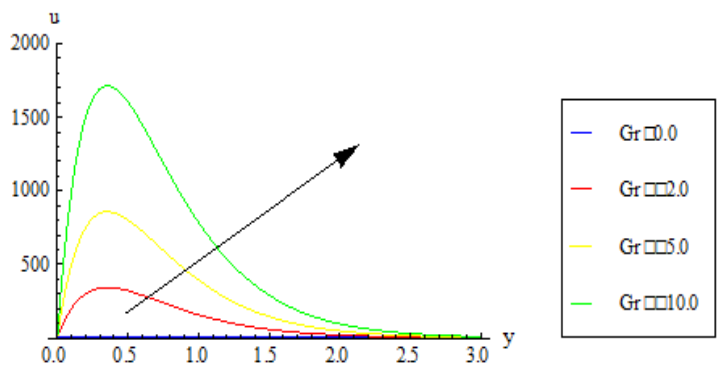

Figure (6) Effect of $\mathrm{Gr}$ on the velocity profile with $\operatorname{Pr}=0.7, \mathrm{M}=2.0, \mathrm{Q}=2.0, \mathrm{Ra}=2.5$
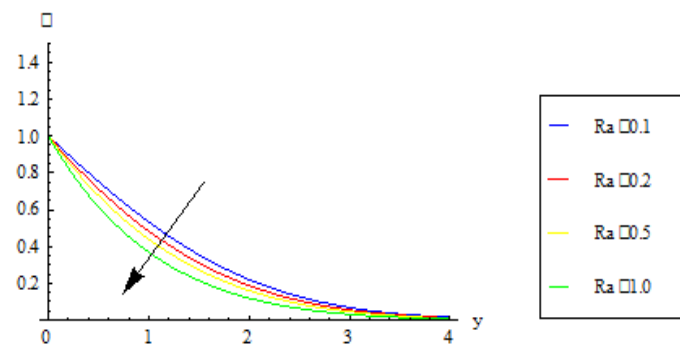

Figure (8) Effect of Q on the temperature with Ra $=0.5, \mathrm{M}=2.0, \mathrm{Gr}=5.0, \mathrm{~K}=8.0$

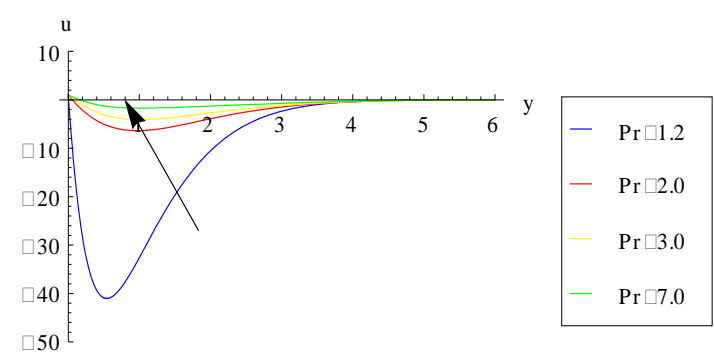

Figure (3) Effect of Pr on temperature profile
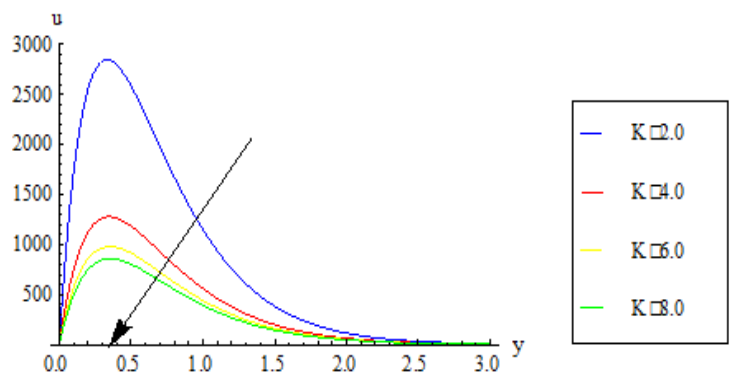

Figure (5) Effect of $\mathrm{K}$ on velocity profiles with $\mathrm{Ra}$ $=0.5, \mathrm{M}=2.0, \mathrm{Q}=2.0, \operatorname{Pr}=0.7$

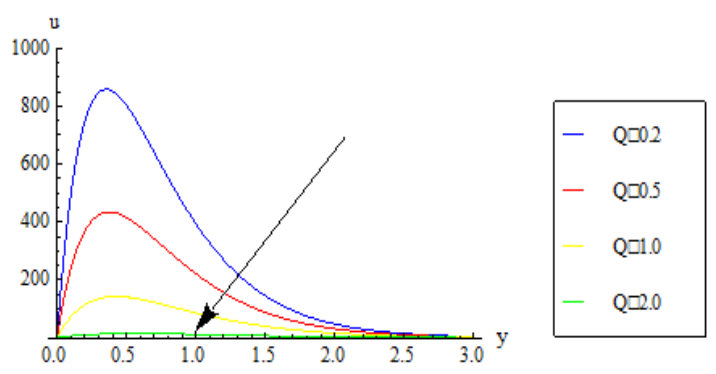

Figure (7) Effect of $\mathrm{Q}$ on the velocity with $\mathrm{Ra}=$ $0.5, \mathrm{M}=2.0, \mathrm{Gr}=5.0, \mathrm{k}=8.0$

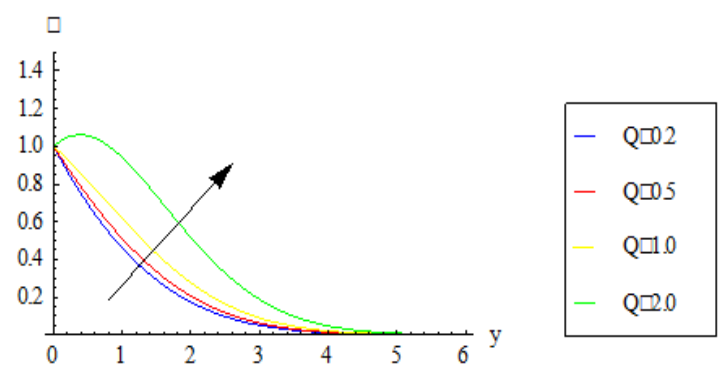

Figure(9) Effect of Ra on temperature $\mathrm{M}=1.5, \mathrm{~K}$ $=2.0, \mathrm{Q}=0.2$ 

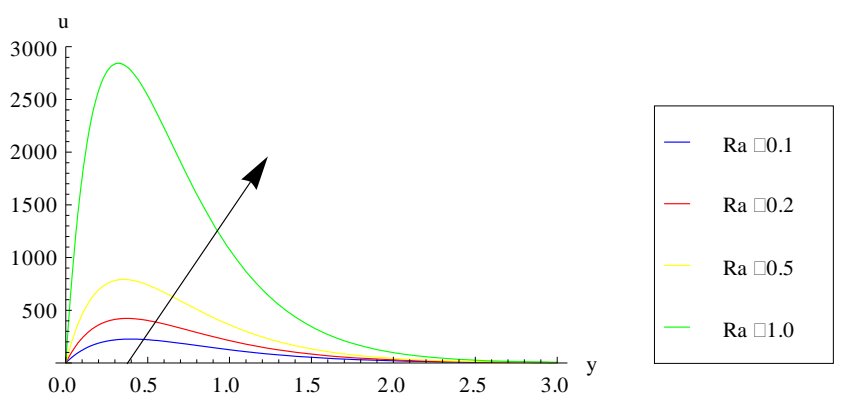

Figure (10) Effect of Ra on velocity with $\mathrm{M}=1.5, \mathrm{~K}=2.0, \mathrm{Q}=0.2, \mathrm{Gr}<0$

In Fig. 5, the effect of the permeability parameter $K$ is shown to cause a decrease on the velocity profile as $K$ increases.

Also Fig. 6 depicts the effect of the Grashof no. $G r$, on the velocity profile. There is a drastic increase on the velocity as $\mathrm{Gr}$ decreases.

Fig. 7 and 8 display the effect of the heat source parameter $Q$ on both the velocity and temperature respectively. It is observed that as $Q$ increases the velocity profile $u$ decreases, while there is an increase in the temperature. The effect of the radiation parameter, $R a$, is shown in Fig. 9 and for the temperature and velocity profiles respectively. Increase in $R a$, gives a decrease in the temperature but an increase in the velocity.

\section{Conclusion}

This problem is a two dimensional unsteady, laminar free convection flow in the presence of and radiation uniform magnetic field applied normal to the flow. The governing equations were normalized and transformed into ordinary differential equations and solved using the Laplace transform technique. The obtained results for velocity and temperature are represented graphically for the various parameters, and were also found to be in good agreement with previous works in the literature. Thus the conclusion on the study are:

1. On increasing the Prandtl number there is a decrease in the temperature while there is an increase in the temperature as the heat flux parameter increases.

2. Also observed that due to the free convection currents for $G r<0$, there is a drastic increase in the velocity field.

The results which have been derived analytically are in good comparison with the results obtained numerically. It is hoped that this can be extended to the case of mass transfer in the presence of radiation for other fluids other than our ait.

\section{Nomenclature}

$c_{p}=$ Specific heat at constant pressure

$q_{r}=$ Radiative flux parameter

$Q=$ Heat source parameter

$k=$ Thermal conductivity

$K=$ Permeability of the porous medium

$G r=$ Grashofs number

$\operatorname{Pr}=$ Prandlt number

$R_{a}=$ Thermal radiation parameter

$M=$ Magnetic parameter

$g=$ Gravitational acceleration

$u, v=$ velocity components

$T=$ Dimensional temperature

$x, y=$ Cartesian coordinates

\section{Symbols}

$\sigma=$ Electrical conductivity

$\gamma=$ Kinematic viscosity

$\tau=$ Skin friction

$\beta=$ coefficient of volume

$\rho=$ Density of your temperature

$\Theta=$ Dimensionless temperature 


\section{Subscript}

$w=$ wall condition

$\infty=$ free stream condition

\section{References}

[1] S. Ostrach, An analysis of laminar free convection flow and heat transfer along a flat plate parallel to the direction of the generating body force, NACA Report 1111, 1952

[2] V. J.Rossow, On flow of electrically conducting fluids over a flat plate in the presence of transverse magnetic field, NACA TN $1957,3971$.

[3] V. M.Soundalgekar, Viscous dissipation effects on unsteady free convective flow past an infinite vertical porous plate with constant suction, International Journal of Heat and Mass transfer 15,1971, $1253-1261$.

[4] B. Gerbhart and L. Pera,The nature of vertical natural convection flow from the combined buoyancy effects on thermal and mass diffusion, International Journal of Heat and Mass transfer 14, 1971, $2024-2050$.

[5] A. R.Bestman,Natural convection boundary later with suction and mass transfer in a porous medium, International Journal of Energy Research 14, $280-296$.

[6] J. C. Geapeau and R.Clarksean,Similarity solutions of natural convection with internal heat generation, Journal of Heat and Mass transfer 119, 1997, $183-185$.

[7] M.A. Hosain andR. A. Began,Effect of mass transfer and free convection on the flow past a vertical plate. ASME Journal of Heat Transfer, 106, 1984, $664-668$.

[8] A. J. Chamkha and A. A. Khaled,Similarity solution for hydromagnetic mixed convection heat and mass transfer for Hiemenz flow through porous media, International Journal of Mechanics Heat fluid flow 10(1), 2000, $94-115$.

[9] A. K. Singh andN. P. Singh,Heat and mass transfer in MHD flow of a viscous fluid past a vertical plate under oscilliating suction velocity, Indian Journal of Pure and Applied Mathematics 2003, 429 - 442.

[10] P. R. Sharma and G. Singh,Unsteady MHD free convective flow and heat transfer along a vertical porous with variable suction and internal heat generation, International Journal of Applied Math and Mechanics 4(5), 2008, $1-8$.

[11] B. Pullepu and A. J. Chamkha, Transient laminar MHD free convective flow past a vertical core with non-uniform surface heat flux, Nonlinear Analysis Modelling and Control 14(A), 2009,489 - 503.

[12] R. Grief, I. S. Habib and L. C. Lin, Laminar convection of a radiating gas in a vertical channel, Journal of Fluid Mechanics 45, $1971,513-520$

[13] A. C. Cogley, W. G. Vincenti and S. E. Gill, Differential approximation for radiative transfer in a non-gray gas near equilibrium, AIAA Journal 6, 1968, 551-553.

[14] H. S. Takhar, R. S. Reddy Gorla and V. M. Soundalgekar, Radiation effects on MHD free convection flow of a gas past a semiinfinite vertical place, International Journal International Joutnal Num. Mech. Heat Fluid flow, 6(2), 1996,77 - 83.

[15] A. J. Chamkha, H. S. Takhar and V. M. Soundalgekar, Radiation effect on free convection flow past a semi-infinite vertical plate with mass transfer, Chemical Engineering Journal 84, 2001, 335 - 342.

[16] B. Shanker, B. P. Reddy and J. A. Rao, Radiation and mass transfer in unsteady MHD free convective fluid flow embedded in a porous medium with heat generation/absorption, Indian Journal of Pure and Applied Physics 48, 2010, 157 - 165.

[17] P. Mebine and E. M Adigio, Unsteady free convection flow with thermal radiation past a vertical porous plate with Newtonian heating, Turkish Journal of Physics, 33, 2009, 109 - 119 .

[18] S. Ahmed and D. Kalita, Laplace technique on magnetohydrodynamic radiating and chemically reacting fluid over an infinite vertical surface, International Journal of Science and Technology 1(5), 1012.

[19] B. M. Abramovitz and I. A. StegunHandbook of Mathematical functions, (Dover New York, 1964). 